\title{
Influences of superconducting fault current limiter (SFCL) on superconducting generator in one- machine double-line system
}

\section{AUTHOR(S):}

Muta, I; Doshita, T; Nakamura, T; Egi, T; Hoshino, T

\section{CITATION:}

Muta, I ... [et al]. Influences of superconducting fault current limiter (SFCL) on

superconducting generator in one-machine double-line system. IEEE TRANSACTIONS ON APPLIED SUPERCONDUCTIVITY 2003, 13(2): 2206-2209

\section{ISSUE DATE:}

2003-06

URL:

http://hdl.handle.net/2433/39951

\section{RIGHT:}

(c)2003 IEEE. Personal use of this material is permitted. However, permission to reprint/republish this material for advertising or promotional purposes or for creating new collective works for resale or redistribution to servers or lists, or to reuse any copyrighted component of this work in other works must be obtained from the IEEE. 


\title{
Influences of Superconducting Fault Current Limiter (SFCL) on Superconducting Generator in One-Machine Double-Line System
}

\author{
Itsuya Muta, Member, IEEE, Takeyuki Doshita, Taketsune Nakamura, Toshiaki Egi, and Tsutomu Hoshino
}

\begin{abstract}
Analysis of superconducting generator in single machine double-line infinite-bus transmission system equipped with SFCL's has been performed by use of EMTDC/PSCAD. When some faults occur in one transmission line, the SFCL in the fault line operates and then the peak fault current can be limited to expected value by use of a function of the SFCL. However, in some cases, the SFCL in the other sound line has been also proved to occasionally operate due to the interaction through each transmission line. Problems due to an installation of the SFCL's in such a model system and the influences on the superconducting generator have been pointed out by simulation analysis.
\end{abstract}

Index Terms-Author, please supply your own keywords or send a blank e-mail to keywords@ieee.org to receive a list of suggested keywords.

\section{INTRODUCTION}

A S IS well known, R\&D on various kinds of superconducting fault current limiters (SFCL's) [1]-[3] is going on worldwide for the purpose to enhance the stability and reliability of highly sophisticated electric power networks. Also a location problem of superconducting AC generators (SCG) with intrinsically high performances has been proposed to cope with earth environmental problems toward reduction of $\mathrm{CO}_{2}$ emission. National R\&D project on SCG in Japan has been going on toward $600 \mathrm{MW}$ class pilot machine, after successfully finishing the 12-years first stage $70 \mathrm{MW}$ class model machine [4] and operating in an actual power grid [5]. Most recently, R\&D on 100 MVA class high- $T c$ superconducting generator has been announced in USA [6].

On the other hand, the location and specifications of the SFCL's have been investigated. Their installation selection problems are of the most importance in order to decide specifications of the SFCL's. Locations to install in main power transmission lines, bank link lines and/or distribution power networks, etc has been considered [7]-[11]. However, there following were not investigated: influences of the SFCL's on alternators and interactions between the SFCL's in electric power networks.

Manuscript received August 5, 2002. This work has been supported in part by Isahaya-Denshi Industry Inc.

I. Muta, T. Nakamura, and T. Hoshino are with the Department of Electrical Engineering, Graduate School of Engineering, Kyoto University (e-mail: muta@kuee.kyoto-u.ac.jp; tk_naka@kuee.kyoto-u.ac.jp; hoshino@kuee. kyoto-u.ac.jp).

T. Doshita is with Mitsui Trade Firm Co. Ltd.

T. Egi is with the Graduate School of Engineering, Kyoto University (e-mail: t-egi@asl.kuee.kyoto-u.ac.jp).

Digital Object Identifier 10.1109/TASC.2003.813037

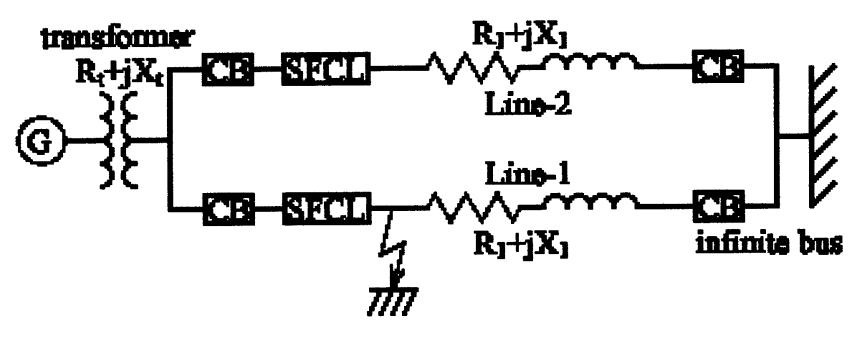

Fig. 1. Single-machine double-line infinite bus system with SFCL.

This paper describes the EMTDC (TM) (which is a general purpose time domain simulation program for simulating power systems transients and its control)/PSCAD (TM) (which is a multipurpose graphical user interface capable of supporting a variety of power system simulation programs) simulation results in terms of SFCLs' influences on a 200 MW class SCG and their interactions, which are installed in single-machine doublepower transmission infinite-bus system. Referring to the basic modeling of the SFCL's in [7], [11], resistive and inductive types were considered under various fault conditions in the above power system, in which a double line is assumed to have the same specification of the SFCL's. Here are presented the simulated results during fault clearing time of only a single line and just after opening the fault line by circuit breaker connected in series with the SFCL. Transients in the SCG designed strongly on SFCLs' parameters. For example, it will be shown that interaction between SFCL's is very important and SFCLs' specifications must be individually adjusted to prevent interactions of SFCL's, and optimal design of the SCG must be modified again.

\section{Simulation System Model}

\section{A. Power System Model}

A simulation system model consists of SCG, step-up transformer, two $100 \mathrm{~km}$ parallel transmission line with circuit breaker, SFCL and infinite bus, is shown in Fig. 1. In the steady state, the rated power (active power $P_{M}=0.8$ pu and reactive power $Q_{M}=0.6 \mathrm{pu}$ ) is assumed to flow through parallel transmission lines. Various kinds of faults have been simulated in line-1 of parallel lines for a fault clearing time of $0.05 \mathrm{~s}$ (3 cycles in $60 \mathrm{~Hz}$ ), changing parameters of the SFCL as stated later. In this paper the simulation results only for the case of sudden 3-phase ground fault and the rated power flows only through the second (nonfault) line after clearing the fault line by the circuit breaker are shown. Other results will be presented in continuous paper. 


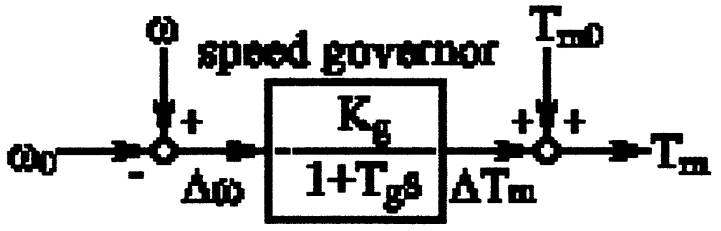

(a)

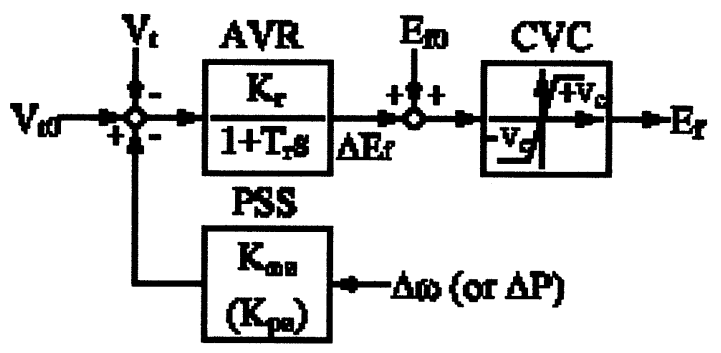

(b)

Fig. 2. Control block diagram of the SCG. (a) GOV block diagram (b) AVR-Exciter block diagram.

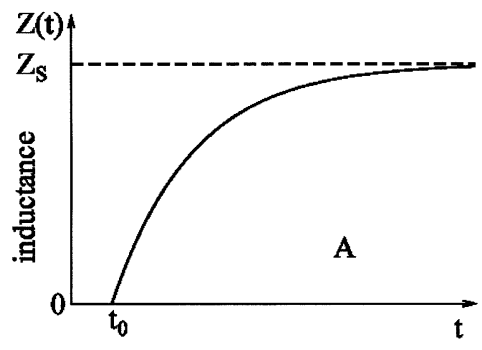

Fig. 3. Time variant impedance model of SFCL.

\section{B. Superconducting Generator (SCG)}

Toward the central part of the SCG, the general structure of SCG consists of magnetic shield, air-cored armature winding, warm damper shield, cold damper/thermal radiation shield, superconducting field winding inside cryogenic vessel, and torque tubes supporting the field winding. In order to control active/ reactive powers and frequency, the SCG is equipped with the simplified speed governor system (GOV: $K_{g}=20 \mathrm{Js}, T_{g}=$ $0.3 \mathrm{~s}$ ) and the automatic voltage regulator (AVR: $K_{r}=20, T_{r}$ $=0.05 \mathrm{~s}$ and $K_{p e}=1.0 \mathrm{~Wb}$ for PSS introduced a feedback input power signal of $\Delta P$ ) with nontraditional (low-voltage, high-current) voltage source as shown in Fig. 2.

\section{SFCL Model}

Two superconducting state to normal conducting state $(\mathrm{S} / \mathrm{N})$ transition type of SFCL's which have same characteristics were applied to parallel transmission lines as shown in Fig. 1. In this paper, no normal state to superconducting state (N/S) recovery of the SFCL during fault clearing time $0.05 \mathrm{~s}$ is assumed to appear. Fig. 3 shows the assumed SFCL model used in simulation [11], providing for resistive and/or inductive functions. Impedance of SFCLs' model is expressed by:

$$
Z(t)=Z_{S}\left[1-\exp \left\{-\left(t-t_{0}\right) / T_{S}\right\}\right]
$$

where $T_{S}$ is time constant to characterize the appearance of the impedance, $t_{0}$ is delay time, and $Z_{S}=R_{S}+j X_{S} . R_{S}$ and $X_{S}$ denote resistive and inductive types, respectively. $t_{0}$ denotes a
TABLE I

SIMULATED CONDITION FOR R-SFCL

\begin{tabular}{cccc}
\hline case & $R_{\mathrm{S}}[\mathrm{pu}]$ & $T_{\mathrm{S}}[\mathrm{ms}]$ & $I_{\mathrm{q}}[\mathrm{pu}]$ \\
\hline 1 & $0.00 .11,0$ & 1.0 & 3.0 \\
2 & 1.0 & 1.010 .050 .0 & 3.0 \\
3 & 1.0 & 1.0 & 1.03 .05 .0 \\
\hline
\end{tabular}

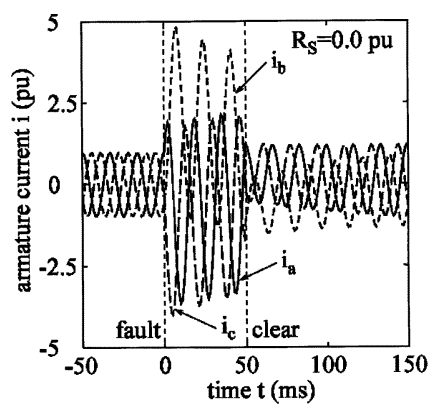

(a)

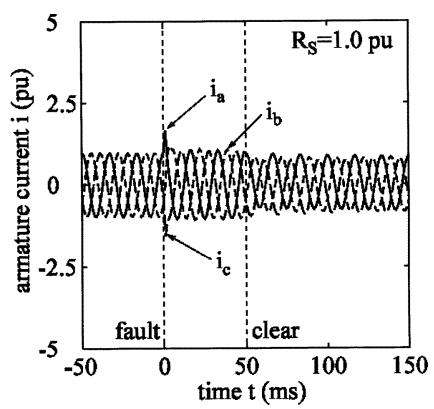

(b)
Fig. 4. Armature current waveform of SCG $\left(T_{S}=1 \mathrm{~ms}, I_{q}=3 \mathrm{pu}\right)$. (a) $R_{S}=0$. pu. (b) $R_{S}=1.0 \mathrm{pu}$.

delay time to initiate $\mathrm{S} / \mathrm{N}$ transition. When fault currents rise up to the expected value $I_{q}, \mathrm{~S} / \mathrm{N}$ transition of the SFCL's is initiated to yield the impedance $Z(t)$.

\section{Simulation Results}

Even in the case of symmetrical fault such as 3-phase ground fault, since an initiation of $\mathrm{S} / \mathrm{N}$ transition for each phase is different, transients in phase current should be noted to be unsymmetrical. The transient peak value of each phase current occasionally happens to be larger than the expected quench value $I_{q}$.

\section{A. Introduction of Resistive Type of SFCL (R-SFCL)}

In the case of a resistive SFCL's (R-SFCL), 3-phase ground fault was first simulated, focusing on each line current and SCG dynamic performances while changing $R_{s c}$ such as $0.0,0.1$, and $1.0 \mathrm{pu}$ and keeping $T_{S}=1.0 \mathrm{~ms}$ and $I_{q}=3.0 \mathrm{pu}$ (case 1). Secondly, while changing $T_{S}=1.0,10.0$, and $50.0 \mathrm{~ms}$ and keeping $R_{S}=1.0$ pu and $I_{q}=3.0 \mathrm{pu}$ (case 2), the same simulation was carried out. Furthermore, while changing $I_{q}=1.0,3.0$, and $5.0 \mathrm{pu}$ and keeping $R_{S}=1.0 \mathrm{pu}$ and $T_{S}=1.0 \mathrm{~ms}$ (case 3 ), the same simulation was done as shown in Table I.

First of all, for $T_{S}=1.0 \mathrm{~ms}$ and $I_{q}=3.0 \mathrm{pu}$ (case 1), transients in armature currents of the SCG dependent on $R_{S}$ and the results are shown in Fig. 4. It should be noted that large value of $R_{S}$ suppresses peak current during fault clearing time and transient currents' variations after clearing time are also moderate. Fig. 5 show transients in transmission lines currents. The introduction of R-SFCL's generally tend to reduce current transients in both lines, but the interference between two lines occasionally happens in case of low $R_{s c}$ and even R-SFCL of the second (nonfault) line was activated.

Next, while changing $T_{S}=1.0,50.0 \mathrm{~ms}$ and keeping $R_{S}=$ $1.0 \mathrm{pu}$ and $I_{q}=3.0 \mathrm{pu}$ (case 2), transients of the armature currents and load angle variations is simulated. In case of $T_{S}=$ $50.0 \mathrm{~ms}$, the R-SFCL of the second (nonfault) line is found to turn to the normal state because there are terminal voltage rise and their currents arise beyond $I_{q}=3.0$ pu. Fig. 6(a) and (b) 


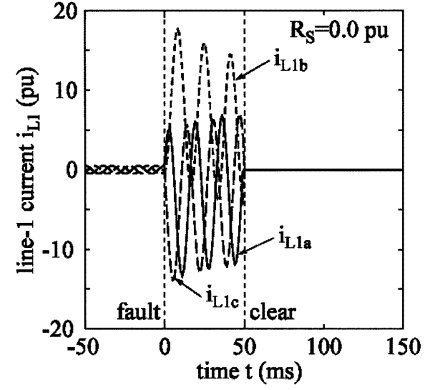

(a)

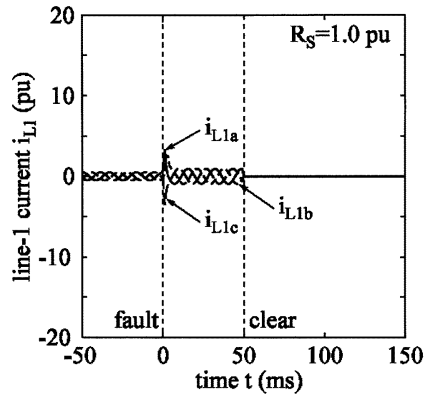

(c)

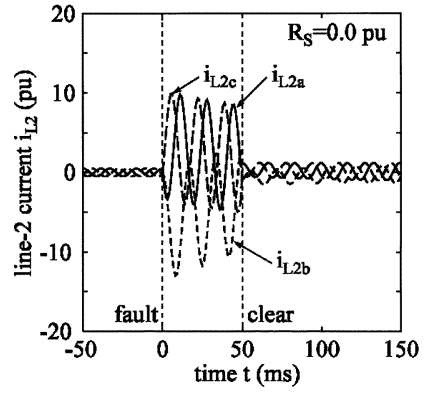

(b)

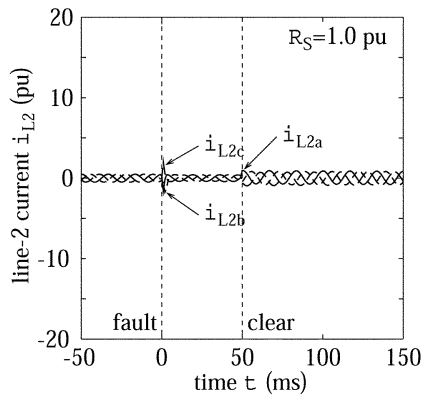

(d)

Fig. 5. Line current waveform ( $T_{S}=1 \mathrm{~ms}, I_{q}=3 \mathrm{pu}$ ). (a) line-1 current waveform. (b) line-2 current waveform.

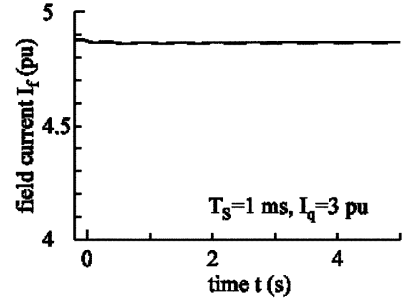

(a)

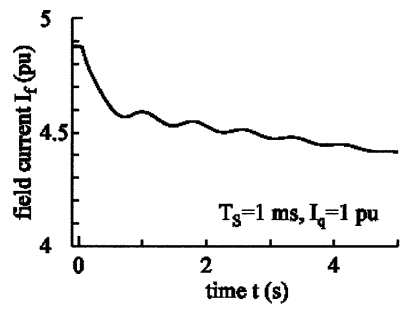

(c)

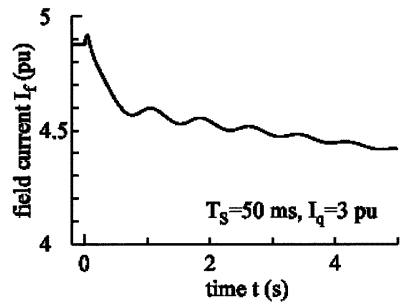

(b)

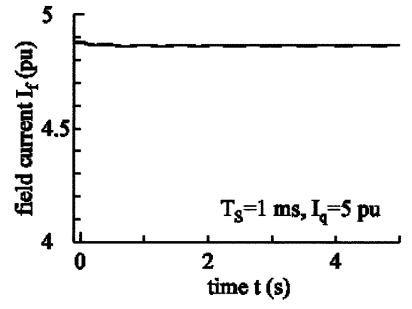

(d)

Fig. 6. Field current waveform of SCG at $R_{S}=1$ pu. (a) $T_{S}=1 \mathrm{~ms}, I_{q}=$ 3 pu. (b) $T_{S}=50 \mathrm{~ms}, I_{q}=3$ pu. (c) $T_{S}=1 \mathrm{~ms}, I_{q}=1$ pu. (d) $T_{S}=1 \mathrm{~ms}$, $I_{q}=5 \mathrm{pu}$.

shows the field current variations of the SCG for cases of $T_{S} x$ $=1.0,50.0 \mathrm{~ms} . T_{S}=1.0 \mathrm{~ms}$ yields little variation.

As the last case, while changing $I_{q}=1.0,5.0 \mathrm{pu}$ and keeping $R_{S}=1.0 \mathrm{pu}$ and $T_{S}=1.0 \mathrm{~ms}$ (case 3 ), the armature current transients are also simulated. In case of $I_{q}=1.0 \mathrm{pu}$, the normal state in R-SFCL's of the second (nonfault) transmission line occurs during fault clearing time as well as changing $R_{S}$ and $T_{S}$. The field current variation of Fig. 6(c) and (d) also demonstrates $\mathrm{S} / \mathrm{N}$ transition of R-SFCL's of the sound transmission line in case of $I_{q}=1.0$ pu. Fig. 4. Armature current waveform of SCG $\left(T_{S}=1 \mathrm{~ms}, I_{q}=3 \mathrm{pu}\right)$.

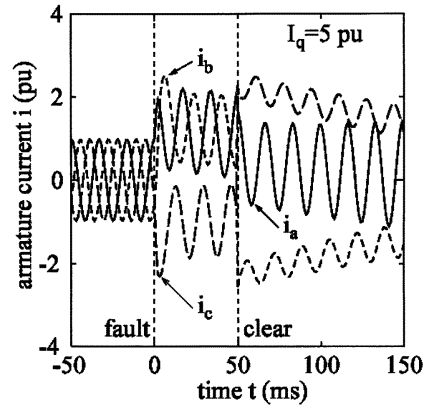

(a)

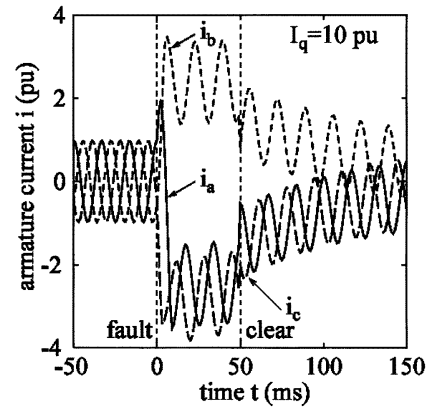

(b)
Fig. 7. Armature current waveform of SCG $\left(X_{S}=1 \mathrm{pu}, T_{S}=1 \mathrm{~ms}\right)$. (a) $I_{q}=5$ pu. (b) $I_{q}=10 \mathrm{pu}$.

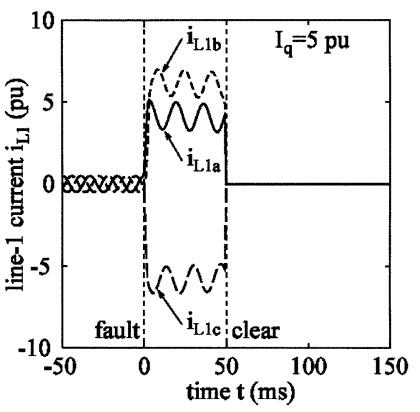

(a)

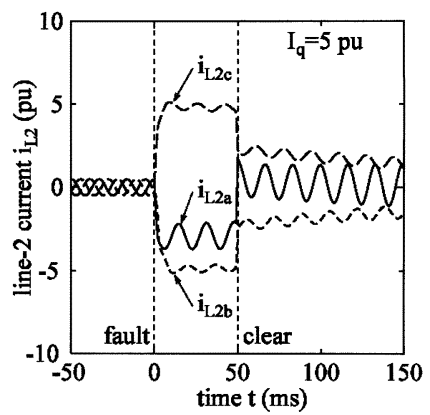

(b)
Fig. 8. Line current waveform $\left(X_{S}=1 \mathrm{pu}, T_{S}=1 \mathrm{~ms}, I_{q}=5 \mathrm{pu}\right)$. (a) line-1 current waveform. (b) line-2 current waveform.

\section{B. Introduction of Inductive Type of SFCL ( $L-S F C L)$}

As parameters of inductive SFCL's (L-SFCL), there is $X_{S}$ as well as $T_{S}$ and $I_{q}$ are used. The system performances, only while changing $I_{q}=5.0,10.0 \mathrm{pu}$, and keeping $X_{S}=1.0 \mathrm{pu}$ and $T_{S}=1.0 \mathrm{~ms}$ are discussed. Fig. 7 illustrates the armature current variations of the SCG. Transients are quite different from those of R-SFCL. Fig. 8 shows the current transients in parallel transmission line. It should be noted that, just like the case of R-SFCL, L-SFCL's of the second (nonfault) transmission line turn to normal state depending on selection of parameters in L-SFCL's, large DC components in current transients appear, resulting in poor damping. This DC components explained by the difference of the time constant $\left(X_{1}+X_{S}\right) / R_{1}$ of the L-SFCL and $X_{1} /\left(R_{1}+R_{S}\right)$ of the R-SFCL.

\section{Comparison of R-SFCL and L-SFCL}

Introduction of both R- and L-SFCL's has been proved to limit fault currents to the expected value by appropriate selection of parameters. However, it is as found interference between SFCL's installed in parallel transmission lines occurred. For example, R-SFCL's of the second (nonfault) line do not cause $\mathrm{S} / \mathrm{N}$ transition for case of larger than about $I_{q}=3.0 \mathrm{pu}$, while L-SFCL's cause S/N transition for case of smaller than about $I_{q}=8.0 \mathrm{pu}$. If moderate $I_{q}$ is desirable, then R-SFCL's have some advantages over L-SFCL's. In addition, R-SFCL's do not cause large disturbances in the SCG armature and the transmission line currents without large DC components during fault and after fault clearing time. On the contrary, in case of L-SFCL's, current transients in the SCG armature and the transmission line currents have very slow damping of large DC components, but rapidly limiting $\mathrm{AC}$ components. 
This simulation study was done just for a simple power system model. Needless to say, case-by-case studies are much required for the introduction and location selection of $\mathrm{R}$ - and L-SFCL's in various power networks.

\section{CONCLUSION}

In this paper, only a simple electric power system with a superconducting generator has been investigated by using EMTDC/PSCAD. SFCLs' characteristics are modeled as exponentially time varying appearance of their impedances, which can define R- and L-SFCL's respectively.

In case of R-SFCL's, fault currents are greatly limited when $R_{S}$ is large and the $T_{S}$ is small, and transients inside the SCG are effectively limited. L-SFCL's are demonstrated to greatly reduce AC components of transient currents. For case of both type of SFCL's, a selection of $I_{q}$ is very important. When its value is too large, current limiting capability is not enough. When its value is too small, SFCL installed in the other (nonfault) power line is shown to be activated occasionally. There is a high probability that black-out may be extending over wide area of power networks, due to interaction in connection with location selection of SFCL's.

\section{REFERENCES}

[1] S. Fischer, H. Schmitt, and R. R. Volkmer, "System requirements and test of superconducting fault current limiters," in CIGRE Session 2000, 2000, no. 13-207.
[2] A. Heinrich, J. Muller, A. Hiebl, K. Numssen, and H. Kinder, "Y-Ba-Cu-O thin films as active high power switches," IEEE Trans. on Applied Superconductivity, vol. 11, no. 1, pp. 1952-1955, March 2001.

[3] T. Hoshino, K. M. Salim, M. Nishikawa, I. Muta, and T. Nakamura, "Dc reactor effect on bridge type superconducting fault current limiter during load increasing," IEEE Trans. on Applied Superconductivity, vol. 11, no. 1, pp. 1944-1947, March 2001.

[4] I. Oishi and K. Nishijima, "Summary of the development of a $70 \mathrm{MW}$ class model superconducting generator-R\&D of superconducting for electric power application" (in Japanese), Cryogenic Engineering, vol. 36, no. 3, pp. 219-227, March 2001.

[5] K. Hasegawa and Y. Imai, "Field test of $70 \mathrm{MW}$ class superconducting generator" (in Japanese), Cryogenic Engineering, vol. 36, no. 3, pp. 256-261, March 2001.

[6] "Design and Develop a 100 MVA superconducting generator," DOE News, April 2001. [Online]. Available: http://www.energy.gov.

[7] N. Hayakawa, H. Kagawa, and H. Okubo, "A system study on superconducting fault current limiting transformer (SFCLT) with the functions of fault current suppression and system stability improvement," IEEE Trans. on Applied Superconductivity, vol. 11, no. 1, pp. 1936-1939, March 2001.

[8] M. Sjostrom and D. Politano, "Technical and economical impacts on a power system by introducing an HTS FCL," IEEE Trans. on Applied Superconductivity, vol. 11, no. 1, pp. 2042-2045, March 2001.

[9] M. Nagata, K. Tanaka, and H. Taniguchi, "FCL location selection in large scale power system," IEEE Trans. on Applied Superconductivity, vol. 11, no. 1, pp. 2489-2494, March 2001

[10] W. Paul et al., "Superconducting fault current limiter applications, technical and economical benefits, simulations and test results," in CIGRE Session 2000, 2000, no. 13-201.

[11] Yu.-H Guo, Y. Yokomizu, T. Matsumura, and H. Fujita, "Difference of effect of superconducting fault current limiter introduced into electric power system due to resistive-type, reactive-type and their introduction location" (in Japanese), Trans. IEE Japan, vol. 120-B, no. 6, pp. 791-800, June 2000. 